- Additional figures are published online only. To view these files please visit the journal online (http://jnnp.bmi. com)

${ }^{1}$ Cognitive Neurology Section, Institute of Neuroscience \& Medicine (INM-3), Research Centre Jülich, Jülich, Germany ${ }^{2}$ Neurological Rehabilitation Centre Godeshöhe, Bonn, Germany

${ }^{3}$ Department of Neurology, University Hospital Cologne, Cologne, Germany

\section{Correspondence to} Dr Simone Vossel, Cognitive Neurology Section, Institute of Neuroscience and Medicine (INM-3), Research Centre Jülich, Leo-Brandt-Str 5, 52425 Jülich, Germany;

s.vossel@fz-juelich.de

Received 14 July 2010 Revised 6 November 2010 Accepted 27 November 2010 Published Online First 17 January 2011

\title{
Visual extinction in relation to visuospatial neglect after right-hemispheric stroke: quantitative assessment and statistical lesion-symptom mapping
}

\author{
S Vossel, ${ }_{1}^{1}$ P Eschenbeck, ${ }^{1}$ P H Weiss, ${ }^{1,3}$ R Weidner, ${ }^{1}$ J Saliger, ${ }^{2}$ H Karbe, ${ }^{2}$ \\ G R Fink ${ }^{1,3}$
}

\begin{abstract}
Background Visual neglect and extinction are two common neurological syndromes in patients with righthemispheric brain damage. Whether and how these two syndromes are associated or share common neural substrates is still a matter of debate.

Methods To address these issues, the authors investigated 56 patients with right-hemispheric stroke with a novel diagnostic test to detect extinction and neglect. In this computerised task, subjects had to respond to target stimuli in uni- and bilateral stimulation conditions with detection probabilities being assessed. A cluster-analytical approach identified 18 patients with neglect and 13 patients with extinction. Statistical lesion-symptom mapping analyses with measures for extinction and neglect were performed.
\end{abstract}

Results Extinction and neglect co-occurred in a subset of patients but were also observed independently from each other, thereby constituting a double dissociation. Lesions within the right inferior parietal cortex were significantly associated with the severity of visual extinction. Visuospatial neglect was related to damage of fronto-parietal brain regions, with parieto-occipital areas affecting line bisection and dorsal fronto-parietal areas affecting cancellation task performance, respectively. Conclusion Quantifying lesion-induced symptoms with this novel paradigm shows that extinction and neglect are dissociable syndromes in patients with righthemispheric stroke. Furthermore, extinction and neglect can be related to differential neural substrates, with extinction being related to focal brain damage within the right inferior parietal cortex.

\section{INTRODUCTION}

Damage to the right cerebral hemisphere frequently results in lateralised disruptions of spatial awareness which cannot be explained by a primary sensory deficit alone. While visuospatial neglect is characterised by a general failure to respond to stimuli in contralesional space, ${ }^{1}$ visual extinction describes a selective impairment of responding to a contralesional stimulus when an ipsilesional stimulus is presented simultaneously. Given the apparent similarity of the two disorders, it has been speculated that the two syndromes are related and share common pathomechanisms, such as an imbalance in hemispheric competition. ${ }^{2-4}$

For instance, patients with neglect may show extinction-like behaviour when they need to disengage their attention from the ipsi- to the contralesional side of space. ${ }^{5-7}$ This becomes manifest in a disproportionate slowing of response times to contralesional targets preceded by invalid (ie, ipsilaterally directing) cues. Although it remains unclear whether disengagement deficits also contribute to the phenomenon of extinction, this emphasises the close relationship between both disorders. Extinction is sometimes considered as a mild form of neglect which may characterise the residual state after recovery from neglect. ${ }^{8}$ The latter, however, may be due at least in part to the fact that extinction may only become obvious after recovery from spatial neglect, since testing for extinction can be difficult when severe neglect is present.

However, extinction has also been dissociated from neglect on behavioural and neuroanatomical grounds. Besides first preliminary evidence for different underlying neuroanatomical substrates with extinction being associated with damage to the temporo-parietal junction ${ }^{9}$ or subcortical areas, ${ }^{10}$ it has been shown that neglect and extinction can occur independently from each other, that is, that there may be a double dissociation between both syndromes. ${ }^{11-13}$

Contrary to the standardised neuropsychological assessment of neglect, extinction is typically examined with a simple confrontation technique. A patient who fails to report the examiner's left finger movement in the bilateral rather than in the unilateral stimulation condition is diagnosed as suffering from extinction. Since this method is not standardised with respect to multiple factors that critically modulate extinction (eg, eccentricity, duration and synchrony of the stimulation ${ }^{814-17}$ ) and lacks fixation control and cut-off scores, its clinical diagnostic value as well as its reliability for cross-sectional and longitudinal examinations of extinction and its neuroanatomical correlates are severely limited. For these reasons, we introduce here a new computerised test for the standardised detection of visual extinction and neglect within the same task. For the first time, we relate a quantitative measure of extinction severity to performance in neglect tests as well as to lesion location by means of statistical voxel-based lesion-symptom mapping (VLSM). ${ }^{18} 19$

\section{METHODS}

\section{Study sample}

All patients and control subjects gave written informed consent before participating in the study. The study was conducted in accordance with the 
ethical principles of the World Medical Association (Declaration of Helsinki) and was approved by the ethics committee of the Medical Faculty of the University of Cologne (approval no: 07214). Seventy-four right-handed subjects participated in this prospective study, including 18 neurologically and psychiatrically healthy subjects (mean age \pm SEM: $52.2 \pm 1.5$ years, seven females). The remaining 56 subjects were patients who had suffered from a right-hemispheric stroke (58.2 \pm 1.1 years, 18 females, mean time poststroke \pm SEM: $112.2 \pm 31.8$ days). These patients were part of a larger study sample of patients with right-hemispheric stroke. ${ }^{20} 21$ Patients with left-hemispheric lesions, signs of dementia (Mini Mental State Examination $\left(\mathrm{MMSE}^{22}\right)$ score $\left.<25\right)$ or psychiatric disorders including alcohol or drug abuse were excluded from the study. Moreover, patients were excluded when they reported not to see the left-sided stimuli with central fixation owing to visual-field defects.

\section{Assessment of visual neglect and extinction}

All patients performed standard neuropsychological paper-andpencil neglect tests from the Behavioural Inattention Test $\left(\mathrm{BIT}^{23}\right)$ including line bisection, line and star cancellation, copying of figures, text reading and clock drawing. While the mean deviation from the true centre of three bisected lines was calculated for the line bisection test (with positive values denoting ipsilesional (rightward) deviations), laterality quotients $(\mathrm{LO}=$ (hits left-hits right)/(hits left + hits right)) were determined for the cancellation tasks. ${ }^{24}$ In addition, the recently proposed 'Centre of Cancellation' (CoC) index ${ }^{25}$ was calculated for the two cancellation tasks.

A novel computerised task was used to test for visual extinction and neglect (see figure 1).

Subjects were asked to fixate at the centre of the screen and to press a button whenever they detected a white square in the display. The following experimental conditions were realised and presented randomly: the target stimulus (square) could be presented either unilaterally, on the left or the right side of space, or bilaterally with a distractor (circle) presented on the opposite side of the screen. Each condition consisted of 20 trials. An equal number of non-target trials were included in which the

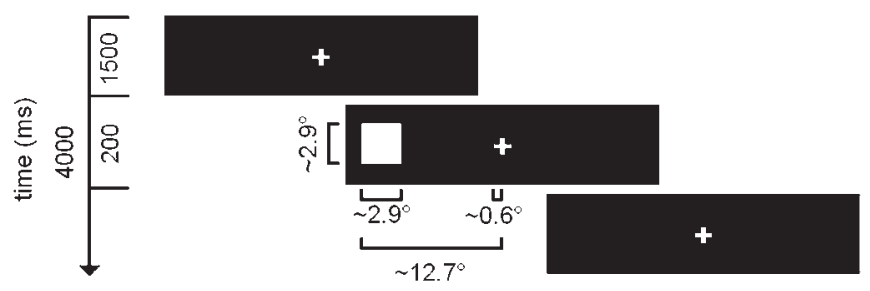

Unilateral Stimulation

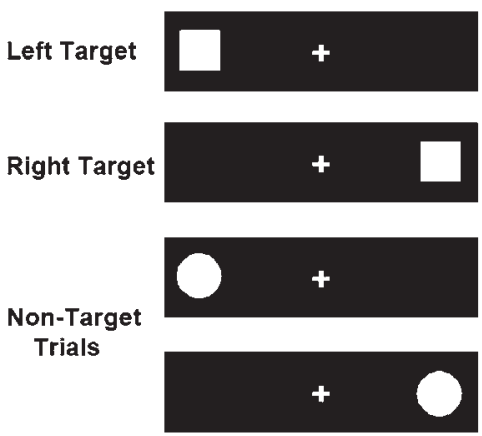

Figure 1 Illustration of the computerised test for visual extinction and neglect. response-irrelevant white circle was presented on the left, right or simultaneously on both sides on the screen. In total, 180 trials were presented. Moreover, 20 'null' trials in which only the fixation cross was shown for $2000 \mathrm{~ms}$ were interspersed with the experimental trials to reduce temporal expectancies.

Detection rates (for targets) as well as false-alarm rates (for non-targets) were used as dependent variables. The detection probability (ie, the percentage of detected targets) in unilateral left trials was used to assess neglect in the computerised task. To obtain a single index of the degree of extinction $\left(\mathrm{I}_{\text {ext }}(\%)\right)$, the following measure was calculated on the basis of the detection probabilities:

$$
\mathrm{I}_{\text {ext }}=\left(\mathrm{P}_{\text {(hit|uni_left })}-\mathrm{P}_{\text {(hit|bi_left })}\right)-\left(\mathrm{P}_{\text {(hit|uni_right) }}-\mathrm{P}_{\text {(hit|bi_right })}\right)
$$

Note that this index implies a relative definition of extinction, since performance is compared between bilateral and unilateral trials without considering the absolute performance in the latter condition. To ensure fixation of the subjects, the investigator was positioned face to face with the subject, so that the occurrence of saccades could be monitored. The investigator signalled the occurrence of a saccade to the presentation computer via a button press, which automatically led to an invalidation of the trial if an eye movement occurred within a period of $1000 \mathrm{~ms}$ before or during the presentation of the response-relevant stimulus. Invalidated trials were automatically appended to the experiment to ensure an equal amount of valid trials across participants. Extinction was also tested with the clinical confrontation technique as described in the Introduction (three passes).

\section{Statistical analyses}

Two-step cluster analyses (maximum of 15 possible clusters, loglikelihood distance estimation, Bayesian Information Criterion for clustering) were performed to identify homogenous subgroups with regard to the performance in the computerised task (unilateral left detection performance and $\mathrm{I}_{\text {ext }}$ ) as well as in the line bisection and cancellation tests. This allowed for the definition of cut-off scores in the respective tests. Cluster analyses of the performance in the computerised task were performed with and without the inclusion of the healthy control subjects. Variables of interest were correlated using the Pearson correlation coefficient. Contingency tables were analysed with $\chi^{2}$ tests to determine if the observed cell frequencies differed significantly from the expected frequencies. Results are reported at a significance level of $p<0.05$.

\section{Lesion analyses}

Brain lesions were confirmed by CT or MRI in all patients. Lesions were drawn manually on a T1-weighted template brain (ch2.nii) with a $5 \mathrm{~mm}$ slice distance using MRIcroN software (http://www.sph.sc.edu/comd/rorden/mricron/). Lesion mapping was performed by SV and rechecked by PHW. Both investigators had to agree jointly on the lesion location and extent, and were blind with regard to the patients' neuropsychological test performance. VLSM was performed with $\mathrm{I}_{\text {ext }}$ and the detection performance in unilateral left trials, as well as with those neglect tests providing quantitative performance measures (ie, the line bisection and cancellation tasks). ${ }^{18}{ }^{19}$ In the VLSM analyses, patients were divided into two groups on a voxelwise basis according to whether a particular voxel in the brain was affected by a patient's lesion or not. Behavioural parameters were then compared between both groups for each voxel, providing voxelwise statistical scores which were tested for significance. Only voxels damaged in at least $5 \%$ of the 
patients were tested. Voxelwise $t$ tests were performed, and results are reported at $p<0.05$ (Bonferroni-corrected for the number of unique lesion patterns). ${ }^{26}$

\section{RESULTS}

Healthy controls performed the computerised task almost without any errors (mean \pm SEM detection rate: unilateral left trials $99.72 \pm 0.28 \%$; unilateral right trials $99.44 \pm 0.38 \%$; bilateral left trials $100 \pm 0 \%$; bilateral right trials $100 \pm 0 \%$; $\mathrm{I}_{\text {ext }}$ $-0.28 \pm 0.28 \%$; false-alarm rates left trials $1.94 \pm 0.92 \%$; right trials $0.83 \pm 0.45 \%$; bilateral trials $1.11 \pm 0.46 \%)$. The frequency of saccade trials was generally very low $(<1 \%$ in all groups or clusters) and was therefore not analysed any further.

The distribution of the detection performance in unilateral left trials and the extinction index $\mathrm{I}_{\text {ext }}$ in the patient group are provided in the supplementary figure S1. Cluster analyses were performed on the total sample of 74 subjects on each of the variables of interest. The analysis of the detection performance in unilateral left trials revealed three subgroups (high performance $(97.24 \pm 0.77 \%$, range: $100-85 \%): n=56$ (18 controls and 38 patients); moderate performance $(62.17 \pm 3.11 \%$, range: $75-45 \%): n=10$ patients; low performance $(8.23 \pm 3.47 \%$, range: 25-0\%): $n=8$ patients). The same clustering results were obtained when the control group was excluded from the analysis. The analysis with $\mathrm{I}_{\text {ext }}$ as classification variable revealed two distinct clusters. While the extinction index amounted to $-2.81 \pm 0.9$ in cluster 1 ( $\mathrm{n}=18$ controls and 43 patients), cluster 2 ( $n=13$ patients) was characterised by high values of $I_{\text {ext }}$ $(34.7 \pm 4.9)$, resulting from a relatively lower detection probability for bi- as for unilaterally presented left targets (see figure 2A). The same clustering results were obtained when the control group was excluded from the analysis or when the eight patients with low detection rates for unilateral left trials were excluded.

Cluster analyses were also performed on the line bisection and cancellation task performance in the patient group. For the two cancellation tasks, the $\mathrm{LO}$ and $\mathrm{CoC}$ indices were highly correlated (line cancellation: $r=-0.950 ; \mathrm{p}<0.001$; star cancellation: $\mathrm{r}=-0.953 ; \mathrm{p}<0.001)$. Moreover, the correlation between both cancellation tests was high (see table 1 below), so that the mean LO of both tests was used in all subsequent analyses. The analyses of the line bisection deviation and the mean $\mathrm{LO}$ of the two cancellation tasks each revealed two separate clusters, with patients with an average ipsilateral line bisection error $\geq 4.17 \mathrm{~cm}$ or an $\mathrm{LQ} \leq-0.5$ being grouped into separate subgroups. Figure 3 depicts the relationship between the extinction index $\mathrm{I}_{\text {ext }}$ and the neglect-related variables (detection rates in unilateral left trials in the computerised task, line bisection deviation and cancellation task LQ).

Contingency tables for extinction and neglect in the different tasks are depicted in table 2. Owing to possible floor effects in
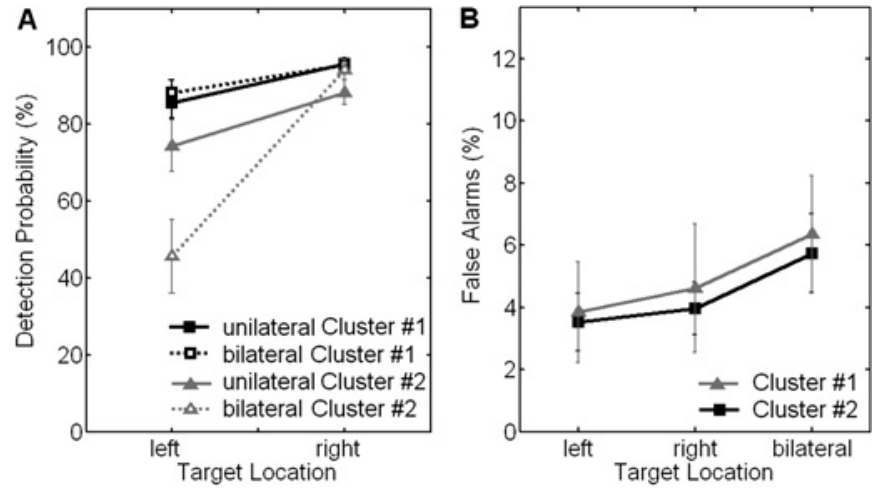

Figure 2 Performance of the two groups revealed by the cluster analysis on the extinction index, $\mathrm{I}_{\mathrm{ext}}$. (A) Detection probabilities in the four different experimental conditions for cluster $1(n=61)$ and cluster 2 $(n=13$, 'extinction cluster'). (B) False-alarm rates for the two clusters. No significant group effects were observed.

patients with low detection performance in unilateral left trials in the computerised task which might constrain a valid diagnosis of extinction, the eight patients with performance $\leq 25 \%$ (cf, figure 3A) were excluded from these analyses.

Table 1 depicts the intercorrelations of the line bisection task, the line and star cancellation tests, the detection performance in unilateral left trials of the computerised task and the extinction index $\mathrm{I}_{\text {ext }}$.

The extinction index $\mathrm{I}_{\text {ext }}$ did not correlate significantly with age or the time poststroke ( $n=48$ patients). The detection probability for unilateral left targets correlated significantly with age $(\mathrm{r}=-0.276 ; \mathrm{p}<0.05 ; \mathrm{n}=56$ patients), but not with the time poststroke.

With the confrontation technique, three of the 13 patients of cluster 2 showed signs of extinction (ie, a failure to detect the left finger movement in the bilateral stimulation condition in at least one out of three passes). Two patients of cluster 1 showed extinction in this test.

The percentage lesion overlap of all 56 patients is provided in supplementary figure S2 to illustrate those brain regions which were most commonly affected by the lesion in the present patient sample. Figure 4 depicts the results of the VLSM analyses relating the extinction index $\mathrm{I}_{\text {ext }}$, the detection performance in unilateral left trials, as well as the performance in the line bisection and cancellation tasks to the critical lesion locations.

The degree of visual extinction (ie, a high extinction index $I_{\text {ext }}$ ) was significantly associated with damage to the right angular gyrus (centre of gravity for maximal significance: $x=52$, $y=-72, z=33$ Montreal Neurological Institute coordinate space; $\mathrm{t}=-5.37$ ) and damage to separate foci in the underlying white matter. The detection probability for unilateral left targets was

Table 1 Pearson correlation coefficients between neglect-related variables and the extinction index $I_{\text {ext }}$

\begin{tabular}{|c|c|c|c|c|c|}
\hline & $\begin{array}{l}\text { Line cancellation } \\
\text { (laterality quotient) }\end{array}$ & $\begin{array}{l}\text { Star cancellation } \\
\text { (laterality quotient) }\end{array}$ & $\begin{array}{l}\text { Line } \\
\text { bisection (cm) }\end{array}$ & $\begin{array}{l}\text { Detection probability for } \\
\text { unilateral left targets }\end{array}$ & $I_{\text {ext }}$ \\
\hline Line cancellation & 1 & $0.752^{* *}$ & $-0.685^{* *}$ & $0.676^{* *}$ & -0.152 (NS) \\
\hline Star cancellation & & 1 & $-0.799 * *$ & $0.792^{* *}$ & -0.213 (NS) \\
\hline Line bisection & & & 1 & $-0.792^{* *}$ & $0.474^{*}$ \\
\hline $\begin{array}{l}\text { Detection probability for unilateral left } \\
\text { targets }\end{array}$ & & & & 1 & $-0.373^{*}$ \\
\hline$I_{\text {ext }}$ & & & & & 1 \\
\hline
\end{tabular}

Those patients with a low detection performance in the unilateral left condition were excluded from this analysis. Laterality quotient=(hits left-hits right)/(hits left+hits right). Note that negative values indicate fewer hits in contralesional (left) as compared with ipsilesional (right) space. Positive values in the line bisection test indicate ipsilesional (rightward) bisection errors. ${ }^{*} \mathrm{p}<0.01 ;{ }^{* *} \mathrm{p}<0.001$ 

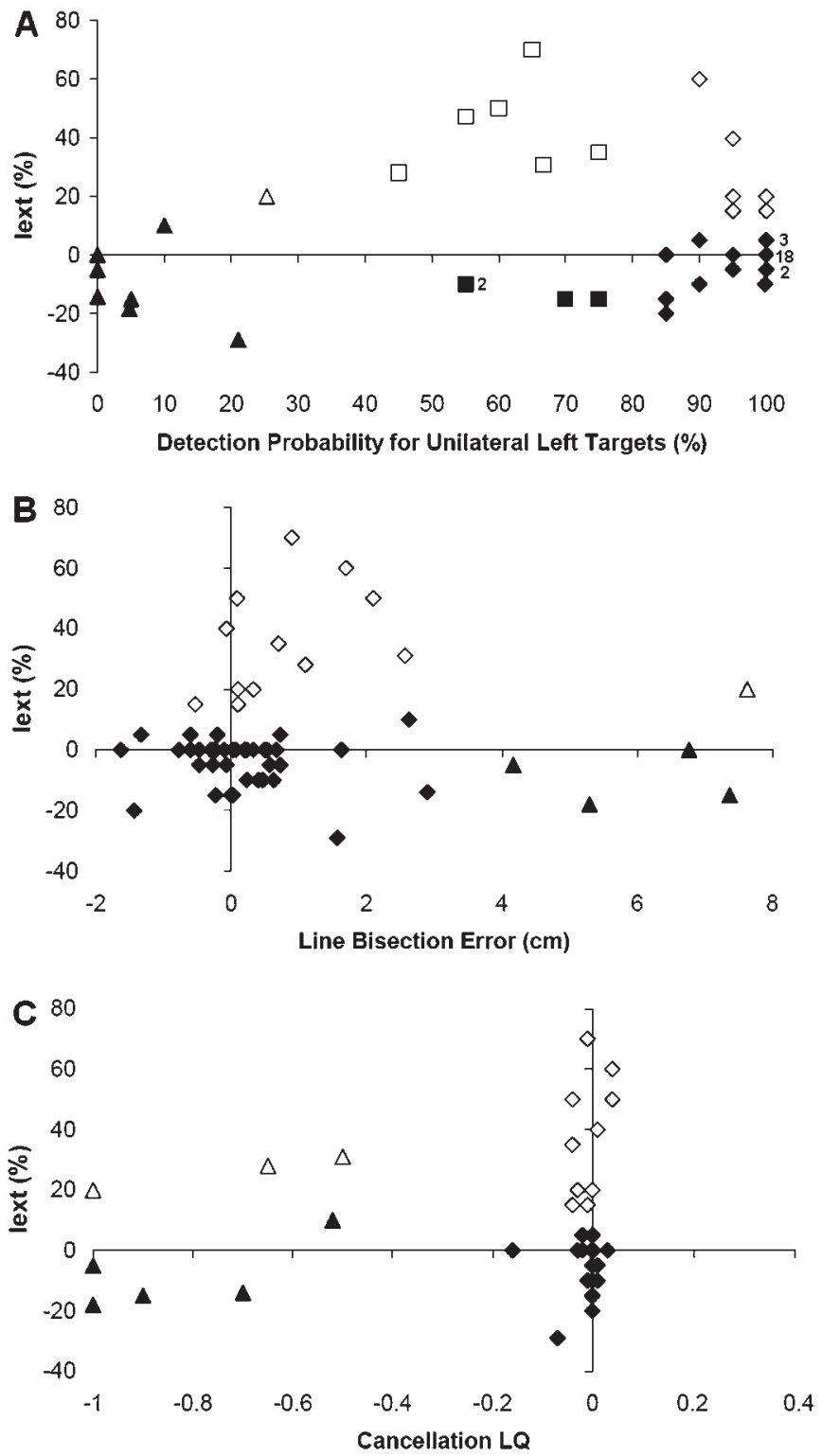

Figure 3 Illustration of the relationship between extinction and neglect as assessed by (A) the detection performance in unilateral left trials in the computerised task, (B) the line bisection test, and (C) cancellation tasks. Different shapes represent different subgroups as revealed by the cluster analyses. Unfilled symbols represent patients of the extinction cluster.

significantly associated with damage to the middle frontal and precentral gyrus and underlying white matter as well as parts of the angular and the middle occipital gyrus. While cancellation performance was related to damage to fronto-parietal areas (including the middle frontal gyrus, pre- and postcentral gyrus and the posterior parietal cortex), line bisection performance was affected by lesions in the middle frontal gyrus and parietooccipital regions as well as with damage to the angular gyrus and the superior parietal lobe.

\section{DISCUSSION}

The present study employed a new computerised test for the assessment of visual extinction and neglect in patients with right-hemispheric stroke. A cluster-analytical approach identified 13 patients who showed a differential decline in the detection of left target stimuli when a right distractor stimulus was simultaneously presented. In some patients, extinction of the left stimulus during bilateral stimulation was associated with the presence of visual neglect symptoms, while in other patients it occurred independently from neglect. Our task can be applied easily in a clinical setting to identify and quantify visual extinction and neglect. Although extinction and neglect frequently co-occur, both can be behaviourally dissociated in patients with right-hemispheric stroke. Given that deficits in visuo-spatial processing predict poor recovery of function after stroke, ${ }^{27}{ }^{28}$ these data strongly suggest that patients with righthemispheric stroke should be assessed for both signs of neglect and extinction. These data moreover show that the severity of visual extinction has a neuroanatomical correlate within the right inferior parietal lobe, while the severity of neglect was more broadly related to damage of fronto-parietal brain areas with additional standard neglect test-specific lesion patterns.

The computerised task - which older healthy control subjects performed almost without any errors-revealed 13 out of 56 patients $(23.2 \%)$ showing extinction in terms of reduced detection rates for bilaterally as compared with unilaterally presented left targets. Moreover, the patient sample could be subdivided into three groups with high (38 out of 56 patients; 67.86\%), moderate (10 patients; $17.86 \%$ ) and low (eight patients; $14.28 \%$ ) detection performance in unilateral left trials signalling the presence of no, moderate or severe neglect, respectively. These prevalence rates for neglect and extinction resemble those reported in a recent study in which neglect was observed in $26.2 \%$ and extinction (assessed with the confrontation technique) in $24.3 \%$ of right-hemisphere patients. ${ }^{11}$ It should be noted, however, that the prevalence of extinction as assessed with the clinical confrontation technique was considerably lower in the present study (see below).

While some patients showed both extinction and neglect, the two syndromes were also observed independently from each other, thereby constituting a double dissociation. However, only one of the patients with severe neglect also showed extinction, which can presumably be attributed to floor effects constraining a valid assessment of extinction in patients with severe neglect. Here, future studies might employ variable (adaptive) target durations to avoid floor effects. ${ }^{29}$ Nonetheless, extinction and neglect still dissociated when patients with severe neglect were excluded from the analyses. Moreover, the finding that the time poststroke was not related to the degree of visual extinction argues against the notion that extinction merely represents a residual form of neglect. Interestingly, some neglect patients without additional evidence for extinction showed higher detection rates in the bilateral than in the unilateral left condition (cf, figure 3A). Indeed, such an 'anti-extinction' phenomenon has already been described in a single case study of a neglect patient and was taken as the first clear evidence for the (neglect without extinction) dissociation between the two syndromes. ${ }^{30}$

The presence or absence of extinction and neglect in the BIT paper-and-pencil tests varied across the different tasks (cf, table 2), presumably due to differences in test difficulty and sensitivity. In the present patient sample, patients with mean line bisection errors $\geq 4 \mathrm{~cm}$ and laterality quotients $\leq-0.5$ were allocated to separate subgroups. Since almost all of these patients also showed severe neglect in the computerised task, they needed to be excluded in the analyses on extinction. Inspection of the remaining patients revealed a positive correlative relationship between line bisection errors and the degree of extinction, while no significant relationship between extinction and cancellation task performance was observed. The latter 
Table 2 Relationship between extinction and neglect-related variables

\begin{tabular}{|c|c|c|c|c|c|}
\hline & & \multicolumn{2}{|c|}{ Extinction } & & \multirow[b]{2}{*}{$\chi^{2}$} \\
\hline & & Present & Absent & & \\
\hline \multirow{2}{*}{$\begin{array}{l}\text { 1. Detection probability for unilateral left } \\
\text { targets }\end{array}$} & Moderate & 6 & 4 & 10 & \multirow[t]{2}{*}{$8.25^{*}$} \\
\hline & High & 6 & 32 & 38 & \\
\hline \multirow{2}{*}{$\begin{array}{l}\text { 2. Neglect in line bisection (mean } \\
\text { rightward deviation } \geq 4.17 \mathrm{~cm} \text { ) }\end{array}$} & Present & 0 & 0 & 0 & \multirow[t]{2}{*}{-} \\
\hline & Absent & 12 & 36 & 48 & \\
\hline \multirow{2}{*}{$\begin{array}{l}\text { 3. Neglect in cancellation (laterality } \\
\text { quotient } \leq-0.50 \text { ) }\end{array}$} & Present & 2 & 0 & 2 & \multirow[t]{2}{*}{$6.26^{*}$} \\
\hline & Absent & 10 & 36 & 46 & \\
\hline \multirow[t]{2}{*}{ 4. Neglect in text reading test } & Present & 3 & 1 & 4 & \multirow[t]{2}{*}{$5.82^{*}$} \\
\hline & Absent & 9 & 35 & 44 & \\
\hline \multirow[t]{2}{*}{ 5. Neglect in figure copying test } & Present & 2 & 2 & 4 & \multirow[t]{2}{*}{ NS } \\
\hline & Absent & 10 & 34 & 44 & \\
\hline \multirow[t]{2}{*}{ 6. Neglect in clock drawing test } & Present & 1 & 1 & 2 & \multirow[t]{2}{*}{ NS } \\
\hline & Absent & 11 & 35 & 46 & \\
\hline \multirow[t]{2}{*}{ Neglect in at least one of the tests $1-6$} & Present & 7 & 8 & 15 & \multirow[t]{2}{*}{$5.46^{*}$} \\
\hline & Absent & 5 & 28 & 33 & \\
\hline
\end{tabular}

Data from 48 patients are depicted, with patients with low detection probability for unilateral left targets in the computerised task being excluded from the analyses. Description of the administration and evaluation of the paper-and-pencil tests: patients were asked to read aloud a newspaper article arranged in three columns, each consisting of two paragraphs. Neglect was regarded to be present if either one of the left paragraphs or the left words in at least two different lines was ignored by the patient. Three drawings (star, cube, flower) were rated for left-sided omissions or size distortions of left elements. A score of $\geq 3$ omissions or distortions for all drawings combined was regarded to indicate neglect. Patients were asked to draw the face of a clock including contour, digits and clock hands on a blank sheet of paper. They were instructed to set the clock hands to the time ' $11: 10$.' The drawn clock was rated according to the following criteria: (1) contralesional omissions/savings of space in the contour, (2) contralesional omissions of numbers, (3) contralesional omissions or ipsilesional misplacement of the clock hands and (4) ipsi- or contralesional compression of the numbers. Neglect was regarded to be present if at least one of these criteria was fulfilled.

${ }^{*} \mathrm{p}<0.05$.

result is at odds with findings by Vuilleumier et $a^{29}$ according to which different measures of extinction in sophisticated computerised tasks were correlated with the number of omissions in a cancellation task. However, the latter study was based on four patients only, and hence, this issue needs further investigation.

The correlation between the extinction assessment with the computerised test and the confrontation technique was low in the present study. Also, fewer patients showed extinction in the clinical confrontation test overall. Beside the already described shortcomings of the latter testing procedure (non-standardised duration, synchrony and eccentricity of the stimulation), it could be speculated that the interaction with the investigator puts the patient in a more alert state for the time of the confrontation test and thus reduces contralesional inattention. However, it has also been suggested that looking at human eyes (as in the clinical confrontation test) may rather impair the ability to perceive a contralesional target in bilateral stimulation conditions in extinction patients. ${ }^{31}$ Furthermore, the longer overall duration of the present computerised task may have put higher demands on sustained attention. Thus, the computerised test might have been more 'tiresome' for the patients, thereby contributing to the discrepant results for the two extinction measures and the increased sensitivity of our new paradigm for detecting extinction. Similarly, a recent study observed that patients with normal performance in the clinical confrontation tests can show severe impairments in detecting left targets in bilateral conditions with increased attentional demands. ${ }^{32} \mathrm{~A}$ further difference from the clinical confrontation test was the use of non-identical (though similar) stimuli in the bilateral conditions of the present task. However, this can hardly explain the discrepancies in sensitivity between both tests, since the use of identical stimuli (ie, two white squares) might rather have further increased extinction severity in the present task (see, eg, Vuilleumier and $\mathrm{Rafal}^{29}$ for a systematic investigation on the effects of shape similarity on extinction).
Previous lesion studies in single cases or small patient samples have suggested that extinction and neglect can be related to different lesion sites. For example, Daffner and colleagues described a patient who showed neglect in cancellation tests but no extinction after damage to the right frontal lobe. ${ }^{33}$ After a subsequent stroke damaging the right parietal cortex, however, the patient's omission rate for left stimuli in bilateral presentations increased from 8 to $83 \%$. Also, in the present study, frontal damage was associated with neglect, as assessed with cancellation tasks rather than with the presence of extinction. However, damage to many different brain regions has been related to neglect, ${ }^{34} 35$ and studies aiming at isolating brain lesions causing extinction show a comparable variability. ${ }^{9} 1036$ Recent methodological lesion-mapping advancements, such as VLSM, now provide a more sensitive approach for relating lesion sites to neurological syndromes and additionally eliminate the appliance of cut-off scores for the definition of patient subgroups which may vary across different studies. VLSM analysis with the extinction index in the present study revealed a significant relationship between the degree of extinction and damage to the right angular gyrus. Thus, when the respective regions within the inferior parietal cortex were affected by the lesion, the ability to detect a left target stimulus in the presence of a right distractor was impaired. These results are only partially consistent with the findings from Karnath and colleagues, who used between-group lesion subtractions to identify the neural basis of extinction. ${ }^{9}$ The authors related extinction to lesions in more ventral temporo-parietal brain areas which, however, also extended into inferior parietal regions. Using the VLSM approach with a similar index assessing lateralised effects of stimulus symmetry in patients with right-hemispheric stroke, a recent study also observed that parts of the intraparietal sulcus extending into the inferior parietal lobe are involved in the processing of competing peripheral stimuli. ${ }^{37}$

Lesions related to visuospatial neglect were located within fronto-parietal areas with further test-specific lesion patterns. 
Figure 4 Results of the voxel-based lesion-symptom mapping (VLSM) analyses. VLSMs were calculated with the extinction index, $I_{\text {ext }}$, as well as with the detection performance in unilateral left trials in the computerised task and the performance in the line bisection and cancellation tasks. Results are shown on axial slices from Montreal Neurological Institute Z-coordinates -42 to 68 at a statistical threshold of $\mathrm{p}<0.05$ (Bonferroni-corrected for the number of unique lesion patterns).

* Those patients with a low detection performance in the unilateral left condition were excluded from this analysis. Almost identical results were obtained when the Centre of Cancellation index was entered into the VLSM analysis on cancellation task performance.

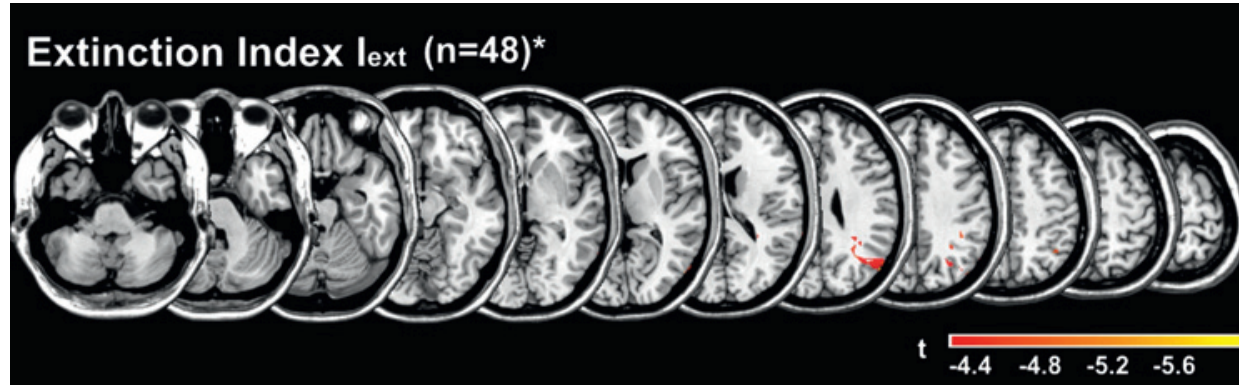

Detection Probability for Unilateral Left Targets $(n=56)$

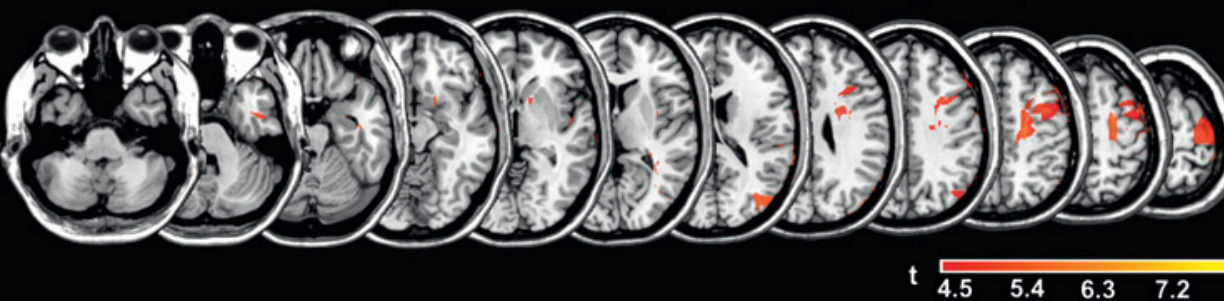

Line Bisection $(n=56)$

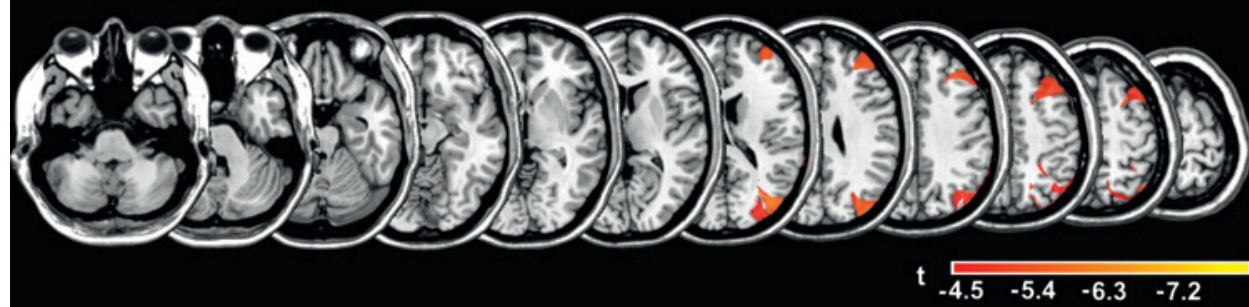

Cancellation Laterality Quotient ( $n=56)$
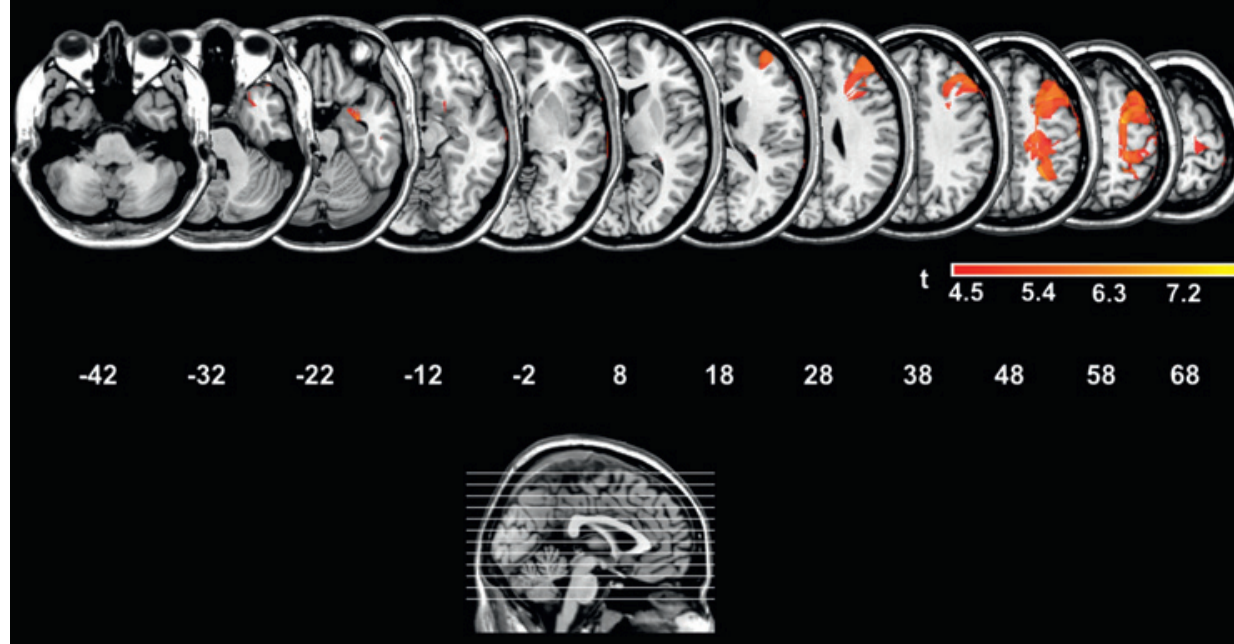

Neglect in the computerised task was related to middle frontal, precentral as well as inferior and occipito-parietal brain areas. Cancellation task performance was affected by damage to dorsal fronto-parietal regions which have been implicated in the voluntary orienting of attention in space. ${ }^{38}$ The finding that line bisection errors were related to a different lesion pattern with an involvement of more posterior parieto-occipital brain regions as well as superior parietal areas is consistent with previous neuroimaging ${ }^{39-42}$ and lesion ${ }^{43}$ data, and highlights the diversity of cognitive demands even within the neuropsychological neglect assessments. For instance, while line bisection may draw upon symmetry judgements, ${ }^{44}$ cancellation tasks require an active exploration of contralesional space and hence voluntary (overt or covert) orienting of attention to left stimuli. These results resemble the findings from a recent VLSM study of different neglect manifestations as identified by a principalcomponent analysis of multiple neuropsychological neglect tests. ${ }^{45}$ Here, the performance in tests with an exploratory/ visuo-motor component was related to lesions within right frontal brain structures, while tests with a perceptive/visuospatial focus (such as the line-bisection task) were rather related to inferior parietal brain damage. These and our results demonstrate that the identification of the neural correlates of extinction and neglect can hardly be achieved by group comparisons of heterogeneous patient groups such as neglect patient samples diagnosed with conglomerate test batteries, 
but requires a more specific inspection and quantification of the cognitive operations assessed with the respective tasks and neuropsychological tests. ${ }^{46}$ This is provided for the domain of visual extinction by the new computerised test presented here. The present task can be easily applied in a clinical setting (with a mean duration for the computerised extinction test of $11.3 \mathrm{~min}$ ) and the standardised assessment can be repeated reliably on separate occasions.

Acknowledgements We thank all patients and control subjects who made this study possible. Moreover, we are grateful to our colleagues from the Cognitive Neurology Section Jülich and the Department of Neurology of the University Hospital, Cologne for valuable support and discussion. We also thank the MaxPlanck Institute for Neurological Research, Cologne, for providing the opportunity to acquire MRI scans of the patients.

\section{Competing interests None.}

Ethics approval Ethics approval was provided by the Ethics Committee of the Medical Faculty of the University of Cologne.

Provenance and peer review Not commissioned; externally peer reviewed.

\section{REFERENCES}

1. Vossel S, Kukolja J, Fink GR. Neurobiology of neglect: implications for new therapeutic strategies. Fortschr Neurol Psychiatr 2010;78:733-45.

2. Kinsbourne M. Hemineglect and hemisphere rivalry. Adv Neurol 1977:18:41-9.

3. Fink GR, Driver J, Rorden C, et al. Neural consequences of competing stimuli in both visual hemifields: a physiological basis for visual extinction. Ann Neurol 2000;47:440-6.

4. Geeraerts S, Lafosse C, Vandenbussche E, et al. A psychophysical study of visual extinction: ipsilesional distractor interference with contralesional orientation thresholds in visual hemineglect patients. Neuropsychologia 2005;43:530-41.

5. Posner MI, Walker JA, Friedrich FJ, et al. Effects of parietal injury on covert orienting of attention. J Neurosci 1984;4:1863-74.

6. Olk B, Hildebrandt $\mathrm{H}$, Kingstone A. Involuntary but not voluntary orienting contributes to a disengage deficit in visual neglect. Cortex 2010;46:1149-64.

7. Losier BJ, Klein RM. A review of the evidence for a disengage deficit following parietal lobe damage. Neurosci Biobehav Rev 2001;25:1-13.

8. Kaplan RF, Cohen RA, Rosengart A, et al. Extinction during time controlled direct retinal stimulation after recovery from right hemispheric stroke. J Neurol Neurosurg Psychiatr 1995;59:534-6.

9. Karnath HO, Himmelbach M, Küker W. The cortical substrates of visual extinction. Neuroreport 2003;14:437-42.

10. Vallar G, Rusconi ML, Bignamini L, et al. Anatomical correlates of visual and tactile extinction in humans: a clinical CT scan study. J Neurol Neurosurg Psychiatr 1994;57:464-70.

11. Becker $\mathbf{E}$, Karnath HO. Incidence of visual extinction after left versus right hemisphere stroke. Stroke 2007;38:3172-4.

12. Cocchini G, Cubelli R, Della Sala S, et al. Neglect without extinction. Cortex 1999;35:285-313.

13. Stone S, Halligan PW, Marshall JC, et al. Unilateral neglect: a common but heterogeneous syndrome. Neurology 1998;50:1902-5.

14. Rorden C, Jelsone L, Simon D, et al. Visual extinction: the effect of temporal and spatial bias. Neuropsychologia 2009;47:321-9.

15. Smania N, Martini MC, Gambina G, et al. The spatial distribution of visual attention in hemineglect and extinction patients. Brain 1998;121:1759-70.

16. Cate A, Behrmann M. Spatial and temporal influences on extinction. Neuropsychologia 2002;40:2206-25.

17. Di Pellegrino G, Basso G, Frassinetti F. Spatial extinction on double asynchronous stimulation. Neuropsychologia 1997;35:1215-23.

18. Bates $\mathbf{E}$, Wilson SM, Saygin AP, et al. Voxel-based lesion-symptom mapping. Nat Neurosci 2003;6:448-50
19. Rorden C, Karnath HO, Bonilha L. Improving lesion-symptom mapping. J Cogn Neurosci 2007; 19:1081-8

20. Eschenbeck $\mathbf{P}$, Vossel $\mathbf{S}$, Weiss $\mathbf{P H}$, et al. Testing for neglect in right-hemispheric stroke patients using a new assessment battery based upon standardized activities of daily living (ADL). Neuropsychologia 2010;48:3488-96.

21. Vossel S, Eschenbeck P, Weiss PH, et al. The neural basis of perceptual and response bias in the Landmark Task. Neuropsychologia 2010;48:3949-54.

22. Folstein MF, Folstein SE, McHugh PR. Mini-Mental State: A practical method for grading the cognitive state of patients for the clinician. J Psychiatr Res 1975;12:189-98

23. Wilson BA, Cockburn J, Halligan P. Behavioural Inattention Test (Bit). Titchfield, UK Thames Valley Test Company, 1987.

24. Bartolomeo P, Chokron S. Egocentric frame of reference: its role in spatial bias afte right hemisphere lesions. Neuropsychologia 1999;37:881-94.

25. Rorden C, Karnath HO. A simple measure of neglect severity. Neuropsychologia 2010;48:2758-63.

26. Kimberg DY, Coslett HB, Schwartz MF. Power in voxel-based lesion-symptom mapping. J Cogn Neurosci 2007;19:1067-80.

27. Cherney LR, Halper AS, Kwasnica CM, et al. Recovery of functional status after right hemisphere stroke: relationship with unilateral neglect. Arch Phys Med Rehab 2001;82:322-8.

28. Robertson IH, Halligan PW. Spatial Neglect: a Clinical Handbook for Diagnosis and Treatment. Hove: Psychology Press, 1999.

29. Vuilleumier $\mathbf{P}$, Rafal RD. A systematic study of visual extinction: between- and within-field deficits of attention in hemispatial neglect. Brain 2000;123:1263-79.

30. Goodrich SJ, Ward R. Anti-extinction following unilateral parietal damage. Cogn Neuropsychol 1997;14:595-612.

31. Maravita A, Posteraro L, Husain M, et al. Looking at human eyes affects contralesional stimulus processing after right-hemispheric stroke. Neurology 2007;69:1619-21.

32. Bonato $\mathbf{M}$, Priftis K, Marenzi R, et al. Increased attentional demands impair contralesional space awareness following stroke. Neuropsychologia 2010;78:3934-40.

33. Daffner KR, Ahern GL, Weintraub S, et al. Dissociated neglect behavior following sequential strokes in the right hemisphere. Ann Neurol 1990;28:97-101.

34. Husain M, Rorden C. Non-spatially lateralized mechanisms in hemispatial negect. Nat Rev Neurosci 2003;4:26-36

35. Halligan PW, Fink GR, Marshall JC, et al. Spatial cognition: evidence from visual neglect. Trends Cogn Sci 2003;7:125-33.

36. Hillis AE, Chang $\mathrm{S}$, Heidler-Gary J, et al. Neural correlates of modality-specific spatial extinction. J Cogn Neurosci 2006;18:1889-98.

37. Molenberghs $\mathbf{P}$, Gillebert CR, Peeters R, et al. Convergence between lesionsymptom mapping and functional magnetic resonance imaging of spatially selective attention in the intact brain. $J$ Neurosci 2008;28:3359-73.

38. Corbetta M, Patel G, Shulman GL. The reorienting system of the human brain: from environment to theory of mind. Neuron 2008;58:306-24.

39. Fink GR, Marshall JC, Shah NJ, et al. Line bisection judgements implicate right parietal cortex and cerebellum as assessed by fMRI. Neurology 2000;54:1324-31.

40. Fink GR, Marshall JC, Weiss PH, et al. The neural bases of horizontal and vertical line bisection judgements: an fMRI study of normal volunteers. Neuroimage 2001;14 S59-67.

41. Weiss PH, Marshall JC, Wunderlich G, et al. Neural consequences of acting in near versus far space: a physiological basis for clinical dissociations. Brain 2000;123:2531-41.

42. Weidner R, Fink GR. The neural mechanisms underlying the Müller-Lyer illusion and its interaction with visuospatial judgements. Cereb Cortex 2007;17:878-84.

43. Rorden C, Fruhmann Berger M, Karnath HO. Disturbed line bisection is associated with posterior brain lesions. Brain Res 2006;1080:17-25

44. Fink GR, Marshall JC, Weiss PH, et al. Task instructions influence the cognitive strategies involved in line bisection judgements: evidence from modulated neural mechanisms revealed by fMRI. Neuropsychologia 2002;40:119-30.

45. Verdon V, Schwartz S, Lovblad KO, et al. Neuroanatomy of hemispatial neglect and its functional components: a study using voxel-based lesion-symptom mapping. Brain 2010;133:880-94.

46. Halligan PW, Marshall JC. Left visuo-spatial neglect: a meaningless entity? Cortex 1992:28:525-35 


\section{Visual extinction in relation to visuospatial neglect after right-hemispheric stroke: quantitative assessment and statistical lesion-symptom mapping}

S Vossel, P Eschenbeck, P H Weiss, et al.

J Neurol Neurosurg Psychiatry 2011 82: 862-868 originally published online January 17,2011

doi: 10.1136/jnnp.2010.224261

Updated information and services can be found at:

http:/jnnn.bmj.com/content/82/8/862.full.html

These include:

References This article cites 44 articles, 13 of which can be accessed free at: http:/jinnp.bmj.com/content/82/8/862.full.html\#ref-list-1

Article cited in:

http://jnnp.bmj.com/content/82/8/862.full.html\#related-urls

Email alerting Receive free email alerts when new articles cite this article. Sign up in service the box at the top right corner of the online article.

Topic
Collections

Articles on similar topics can be found in the following collections

Stroke (1186 articles)

Notes

To request permissions go to:

http://group.bmj.com/group/rights-licensing/permissions

To order reprints go to:

http://journals.bmj.com/cgi/reprintform

To subscribe to BMJ go to:

http://group.bmj.com/subscribe/ 